\title{
Fasting Gastrointestinal Tract: Changes in Structure, Functions and Microbiota Milieu
}

\section{PD Gupta}

Former, Director Grade Scientist, Centre for Cellular and Molecular Biology, Hyderabad, India.

Corresponding Author: PD Gupta, Former, Director Grade Scientist, Centre for Cellular and Molecular Biology, Hyderabad, India.

Received date: June 20, 2021; Accepted date: June 21, 2021; Published date: June 28,2021

Citation: P.D. Gupta (2021) Fasting Gastrointestinal Tract: Changes in Structure, Functions and Microbiota Milieu J. Gastroenterology Pancreatology and Hepatobiliary Disorders. 5(3): DOI: 10.31579/2641-5194/037

Copyright: @ 2021, PD Gupta, This is an open access article distributed under the Creative Commons Attribution License, which permits unrestricted use, distribution, and reproduction in any medium, provided the original work is properly cited.

\begin{abstract}
Short and Intermittent fasting brings many structural and functional changes in the GI tract.Now it has been shown that changes are beneficial in terms of postponing the onset of NCDs prolonging the healthage, That might be the reason for Ancient Indian saints todo fasting. These benefits have been described in a series of research papers by us and others. Change in microbiota due to fasting is a recent addition which explains the mechanism of many physiological processes taking place during fasting.Two other parameters namely, effect of long and short term fasting and.sexual dimorphism on microbiota also dealt with in this review.
\end{abstract}

Keywords: gastrointestinal tract: microbiota milieu

\section{Introduction}

The biological clock controls many cyclic functions in the human body and hunger is one of those [1].The gastrointestinal (GI) tube from mouth to anus is lodged in the abdominal cavity involved in digestion of the food. Digestion is the complex process of turning the food into gastric juices, that assimilate withe the blood circulate all along the body. The body uses these juices for energy, growth and cell repair needed for an individual to survive. The digestion process also involves creating waste from the food which we eat to be eliminated. The foods which we eat provide energy to the body in the form of Adenosine triphosphate (ATP) [2]. In addition to digestive enzyme systems, GI secretes hormones like gastrin, secretin which control the different digestive enzymes that are secreted in the stomach and the intestine during the process of digestion and absorption [3].

The third component which gained importance lately is the microbiota; this is essentially a microbial organ that provides metabolic, immunologic, and protective functions for the host. This is also observed that intestinal components always remain in balance, if intra or inter component balance is disterbed one would get sick. [4]. Habitual diet, changes in macronutrient composition, and consumption of nondigestible dietary fibers have all been shown to impact the human GI microbiome. Intriguingly, the impact of diet on the microbiome may be related not only to what humans eat but also to the timing of food consumption [5]. Therefore fasting has a profound effect on the composition and function of the enterocytes and microbiota. Emerging preclinical research suggests that gut microbes experience diurnal rhythms, and the health effects of eating patterns, including time-restricted feeding and meal frequency, may be related to the GI microbiota [6].
Dietary restrictions for their promising effects on longevity and healthspan have been adopted by health conscious populations globally, It also affects age-associated diseases and, in clinical studies, promotes beneficial health outcomes. Because long-term compliance with daily calorie restriction has proven problematic in humans, several intermittent fasting regimens, including alternate day fasting and time-restricted feeding, have evolved revealing similar clinical benefits as calorie restriction [7]

\section{Changes in the inner Lining}

During fasting (starvation) there are major changes in the structure, composition, and functions of the microvilli (brush border membrane $(B B M))$ of the intestinal epithelial cells.[8,9] The surface area of the BBM becomes at least one and half times per cell compared to normal fed intestine border membrane (BBM)[10]. The density of IMPs and filipinsterol complexes (FSCs) decrease considerably during starvation [11]. Biochemical estimations show Alterations in the lipid and fatty acid composition of BBM, a decrease in the levels of cholesterol and proteins with respect to phospholipids, sphingomyelin/phosphatidylcholine $(\mathrm{mol} / \mathrm{mol})$, protein/lipid (w/w), and free fatty ()acids (w/w) decreased whereas the total phospholipid $(\mathrm{w} / \mathrm{w})$ ratio and the double-bond index increased. Analyses of fatty acids showed higher percentages of stearic and arachidonic acids whereas oleic and linoleic acids decreased under starvation [12] On refeeding, these changes were restored to well-fed levels[13]. The change in membrane state under starvation is associated with alterations in the lipid and fatty acid composition of BBM and may be responsible for functional changes that occur under nutritional stress.During starvation which is in agreement with morphological findings. $[8,11,12,13]$ 
Energy goes towards healing our bodies instead of digesting food. Fasting also allows for the body's enzyme system to focus on detoxifying and breaking down toxins in the body quickly and efficiently without the job of heavy food digestion $[14,15]$.

\section{Microbiota}

There is a fairly good knowledge we have towards the cellular and physiological changes for the fasting intestine and benefits of fasting; however, relatively little knowledge has been acquired of the impact of these various fasting protocols on the gut microbiome (GM). Fasting gut may beneficially alter the composition and function of a welfed gut microbiota.It was known that non-communicable diseases such as obesity, diabetes, cancer and neurological and gastrointestinal inflammatory diseases(NCDs) could be controlled by dietary restrictions and/or intermittent or short term fasting. The new insights that the gut microbiota that was reshuffled during fasting has positive influence on host metabolism, gut barrier permeability, brain functions, and subsequently, postpone the onset of NCDs prolonging the health [16].

Most of the time western diet can promote deleterious changes in the GM $[17,18]$. These microbiota interfere in lipid and glucose metabolism. In addition to this the well fed intestinal microbiota weakens immune responses [19]. Fasting has been demonstrated to increase the abundance of protective, beneficial microbial families,such as Bifidobacteriaceae, Lactobacillaceae and Akkermanisaceae. The initial findings may be promising for the use of fasting to beneficially influence and alter the gut microbiota.Intermittent fasting might directly affect gut microbial composition, function and interaction with the host [17, 18]. Fasting could have a profound and long-lasting effect on human GIT microbiome and drove individuals to develop a more homogenous GIT microbial community.Ongoing research on the health impact of fasting regimens on GM modulation is warranted and some of its benefits could be mediated by the circadian system [20].

\section{Long and Short term Fasting Effects on microbiota}

Longer periods of fasting appear to reduce total bacterial load, and this is associated with improved gut health and lifespan extension.

We also studied healthy humans to look at the gut microbiome to see how it changes with 2 fasts per week. At the 12-16 hour mark, we saw a dramatic shift in the gut microbiome population after fasting for that period. Certain bacteria are super responsive to fasting, and those tend to be beneficial bacteria.Dietary restriction (DR) decreases body weight, improves health, and extends lifespan. Dietary and pharmacological interventions promote a healthy lifespan by influencing energy intake and circadian rhythms. [21].Short-term fasting (14-22 h) resulted in sexually dimorphic metabolic changes, which were more pronounced during prolonged fasting $(38-72 \mathrm{~h})$

\section{Effects of Sexual dimorphism on microbiota}

Effects of fasting differ between men and women. Women metabolize lipids more during fasting, while men have preference of carbohydrate utilization (22). Plasma glucose concentrations were shown to be lower, while free fatty acids (FFA) and lipolysis were higher in women compared to men after fasting. Mechanisms that can increase lipolytic rates in women include higher total fat mass, enhanced lipolytic sensitivity to epinephrine, and increased activation of $\beta$ adrenergic receptors [23].

\section{References}

1. Gupta P D and Pushkala K (2012) CLock within Us Lambert, Academic Publishing, GmbH \& Co. Germany.
2. Liao DH, Zhao JB, Gregersen H (2009). Gastrointestinal tract modelling in health and disease. World J Gastroenterol. 15 (2): 169-76.;

3. Thomas R P., Mark R. et al. (2003). Role of Gastrointestinal Hormones in the Proliferation of Normal and Neoplastic Tissues, Endocrine Reviews, 24, (5), 571-599.

4. Sawicka, B., Johar, K. and Sood, P.P. and Gupta PD. (2017). Imbalance of gut microbiota induces Cancer. Journal of Cell Tissue Research; 17(2): 6073-6084. 2017

5. Gupta P D Time and its control in the body-, Chapter2- The biological clock In Concepts in Chronopharmacology, Shyam Prakashan, Jaipur, India

6. Kaczmarek JL, Thompson SV, Holscher HD.(2017) Complex interactions of circadian rhythms, eating behaviors, and the gastrointestinal microbiota and their potential impact on health. Nutr Rev.; 75(9):673-682. 2017

7. Gupta. P D (2020)Food Addiction and Intermittent Fasting. J. Addict, Res. 4 (1) 1-f 3.

8. Waheed AA, Gupta PD. (1997) Changes in structural and functional properties of rat intestinal brush border membrane during starvation. Life Sci. 61(25): 2425-33.;

9. Pal M K and Sareesh et al. (2010) Rat everted intestinal segments demonstrate that fasting is a regulator of uptake and metabolism of glucose Indian J Physiol Pharmacol 54(1):89-91

10. Waheed,A.A Toyama, Y Yasusumi F Gupta, P(1998) .Decreased densities of intramembranous particles and cytochemically detectable cholesterol in microvilli of starved rat intestine, Cell Biology International, 22, (3) 177-183

11. Gupta PD, Waheed AA. (1992) Effect of starvation on glucose transport and membrane fluidity in rat intestinal epithelial cells. FEBS Lett.300 (3): 263-267.

12. Waheed, A.A., Yasuzumi, F. \& Gupta, P.D. (1998) Lipid and fatty acid composition of brush border membrane of rat intestine during starvation. Lipids 33, 1093-1097

13. Luciano.L, Gupta PD, et al. Modulation of apoptosis by starvation: morphological and biochemical study of rat intestinal mucosa.

14. Cell death and differentiation 2 (4), 259-266 (1995)

15. Liu, Z., Dai, X., Zhang, H. et al. Gut microbiota mediates intermittent-fasting alleviation of diabetes-induced cognitive impairment. Nat Commun 11, 855.

16. Wilhelmi de Toledo,F, Cesare R F et al. Unravelling the health effects of fasting: a long road from obesity treatment to healthy life span increase and improved cognition, Annals of Medicine, 52:5, 147-161, 2020

17. Rinninella O,Marco Cintoni et al. (2020) Gut Microbiota during Dietary Restrictions: New Insights in Non-Communicable DiseasesMicroorganisms 8(8), 1140,

18. PennisiDec, Elizabeth, et al. (2017). "Extreme Diets Can Quickly Alter Gut Bacteria."

19. David, Lawrence A., et al. (2013). "Diet Rapidly and Reproducibly Alters the Human Gut Microbiome.” Nature News, Nature Publishing Group.

20. Campos-Rodriguez, R., et al. (2014) "Intermittent Fasting Promotes Bacterial Clearance and Intestinal IgA Production in Salmonella Typhimurium-Infected Mice.” Wiley Online Library, John Wiley \& Sons, Ltd, 20

21. Alex E. Mohr, Eric Gumpricht, et al. (2021) Recent advances and health implications of dietary fasting regimens on the gut microbiome.Am J Physiol Gastrointest Liver Physiol. 320(5):G847-G863.

22. Catterson JH, Khericha M,et al. (2018) Short-Term, Intermittent Fasting Induces Long-Lasting Gut Health and TOR-Independent Lifespan Extension. Curr Biol. 28(11):1714-1724.e 
23. Min Y, Ma X, et al. (2019) Sex-specific association between gut microbiome and fat distribution. Nat Commun. ;10(1):2408.
24. Hedrington MS, Davis SN. (2015) Sexual Dimorphism in Glucose and Lipid Metabolism during Fasting, Hypoglycemia, and Exercise. Front Endocrinol (Lausanne).6:61. (c)

This work is licensed under Creative

Commons Attribution 4.0 License

To Submit Your Article Click Here: Submit Manuscript

DOI: $10.31579 / 2641-5194 / 037$
Ready to submit your research? Choose Auctores and benefit from:

* fast, convenient online submission

* rigorous peer review by experienced research in your field

* rapid publication on acceptance

* authors retain copyrights

* unique DOl for all articles

* immediate, unrestricted online access

At Auctores, research is always in progress.

Learn more www.auctoresonline.org/journals/gastroenterologypancreatology-and-hepatobilary-disorders 